\title{
Resultados del Programa Pueblos Mágicos en Tlatlauquitepec, Puebla: una visión de los actores sociales
}

\section{Results of the Magic Towns Program in Tlatlauquitepec, Puebla: a vision of the social actors}

Luz del Carmen Morán-Bravo1; Ricardo Osorio-Gómez;

María de los Ángeles Flores-Aguilar³; Héctor De Sampedro-Poblano ${ }^{4}$

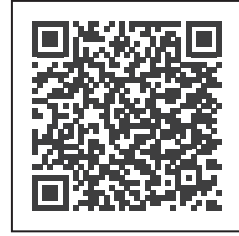

\section{Palabras clave:}

comunidad local; desarrollo económico y social; estadísticas; investigación; marca; pueblo mágico; turismo cultural.

Artículo de investigación científica y tecnológica

Fecha de recepción:

15 de marzo de 2021

Fecha de aprobación:

25 de noviembre de 2021

Fecha de publicación:

22 de diciembre de 2021

Esta publicación se encuentra bajo licencia:

Creative Commons

Reconocimiento-

NoComercial-SinObraDerivada 4.0 Internacional

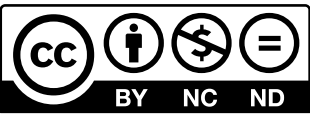

\section{Resumen}

Problemática: la riqueza natural, cultural, histórica y gastronómica de México es una oportunidad de reactivación económica (Sectur, 2020). En 2001 se crea el Programa Pueblos Mágicos, que promueve el desarrollo local de las regiones. Hasta hoy, hay132 reconocidas, entre ellas Tlatlauquitepec, ubicada entre los diez mejores atractivos turísticos de Puebla. Objetivo del estudio: identificar los resultados del programa desde la percepción de los grupos de interés: empresarios, ciudadanía, turistas y autoridades municipales. Materiales y métodos: investigación descriptiva. La información se recopila con una encuesta para cada grupo; para las autoridades, se realiza un censo complementado con entrevistas. Resultados: los habitantes de Tlatlauquitepec conservan prácticas tradicionales de organización y producción agrícola y desarrollan habilidades para atender al turismo. Con el distinti-

Licenciatura en Administración Pública, doctorado en Administración Pública, Universidad Tecnológica de Puebla, México. Icmoran.utpuebla@gmail.co, ORCID: https://orcid.org/0000-0002-7096-2075

2 Licenciatura en Administración Pública, doctorado en Administración Pública, Tecnm - Instituto Tecnológico de Puebla, México. ricardo.osorio@puebla. tecnm.mx, ORCID: https://orcid.org/0000-0002-4478-8333

3 Licenciatura en Diseño Gráfico, doct5rado en Planeación Estratégica y Dirección de Tecnología, Universidad Tecnológica de Tecamachalco, Puebla, México. angelf35@hotmail.com, ORCID: https://orcid.org/0000-0003-2275-3639 4 Licenciatura en Diseño Gráfico, maestría en Tecnología Educativa, Universidad Tecnológica de Puebla, México. hector.desampedro@utpuebla.edu.mx, ORCID: https://orcid.org/0000-0002-9235-9007 


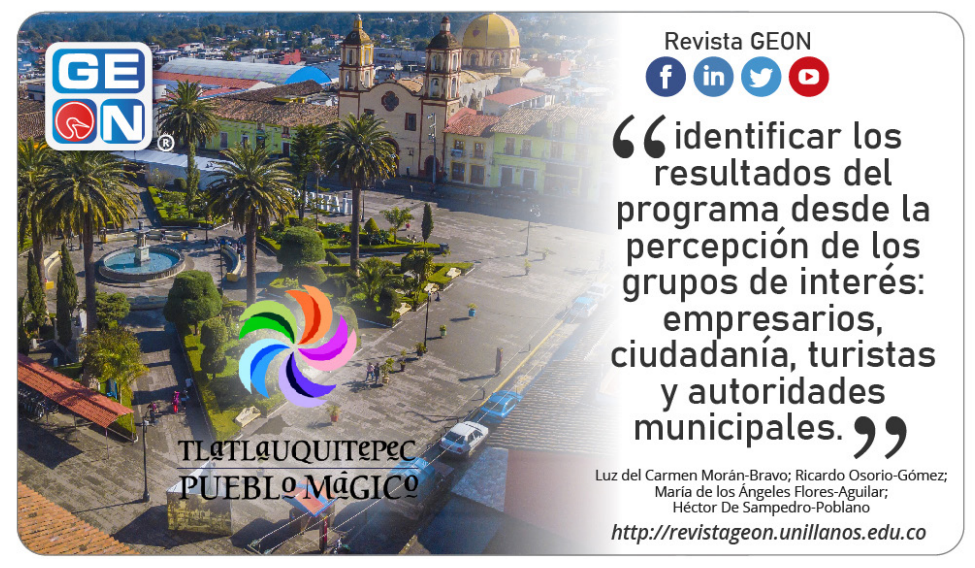

vo de Pueblo Mágico se generaron empleos permanentes y temporales. En 2010 se tenían 4 hoteles y en 2020 estos se incrementaron a 16 (Sectur, Puebla, 2021), propiciando un mayor dinamismo económico con negocios locales. Por otra parte, la afluencia del turismo incrementa la basura, la contaminación y el encarecimiento de los bienes y servicios de la localidad. Discusión: el programa revalora las poblaciones con potencial turístico sin desarrollar (Pérez, 2018) y reactiva la economía, beneficiando a las empresas turísticas. Sin embargo, se incrementan algunos precios, lo que no favorece la economía familiar. Conclusiones: el programa favoreció a algunos sectores empresariales y de la población, pero debe evitar la centralización en la cabecera municipal. Es necesaria la participación de cada grupo de interés. Contribuciones y originalidad: apoyo en la gestión del Plan de Desarrollo Turístico Integral, identificación de fortalezas y oportunidades en los recursos naturales, gastronómicos y culturales, capacitación para mejorar la calidad de los servicios y productos en las comunidades con potencial turístico poco desarrollado, como Oyameles y Mazatepec. Cómo citar este artículo /
Toreference this article:

Morán-Bravo, L. del C., OsorioGómez, R., Flores-Aguilar, M. de los A., \& de Sampedro-Poblano, H. (2021). Resultados del Programa Pueblos Mágicos en Tlatlauquitepec, Puebla: una visión de los actores sociales. Revista GEON (Gestión, Organizaciones Y Negocios), 8(2), e-325. https://doi. org/10.22579/23463910.325
Códigos JEL: Z32 Turismo y desarrollo

Palabras clave: calidad; desarrollo; economía y negocios; población; Pueblo Mágico; turismo.

\section{Abstract}

Problematic: The natural, cultural, historical and gastronomic wealth of Mexico is an opportunity 
Cómo citar este artículo / Toreference this article:

Morán-Bravo, L. del C., OsorioGómez, R., Flores-Aguilar, M. de los A., \& de Sampedro-Poblano, H. (2021). Resultados del Programa Pueblos Mágicos en Tlatlauquitepec, Puebla: una visión de los actores sociales. Revista GEON (Gestión, Organizaciones Y Negocios), 8(2), e-325. https://doi. org/10.22579/23463910.325 for economic reactivation (Sectur, 2020). In 2001 it was created the magical towns program, which promotes the local development of the regions, thanks to this program today there are 132 regions recognized, including Tlatlahuquitepec, located among the ten best tourist attractions in Puebla. Objectives: The objective of the study is to identify the results of the program from the perception of the interest groups: businessmen, citizens, tourists and municipal authorities. Materials and methods: Descriptive research, The information is collected with a survey for each group; for authorities, a census is carried out supplemented with interviews. Results: The residents keep traditional practices of agricultural organization and production, in adittion these develop skills to serve tourism are developed. With the badge, permanent and temporary jobs were created. In 2010 there were 4 hotels and in 2020 it increased to 16 (Sectur, Puebla:2021) a, promoting greater economic dynamism with local businesses. Consequently the influx of tourism increases garbage, pollution and increases the cost of goods and services in the town. Discussion: the program revalues populations with undeveloped tourism potential (Pérez, 2018), reactivates the economy benefiting tourism companies. Prices are increased that do not favor the family economy. Conclusions: the program favored some business sectors and the population, but should avoid centralization in the municipal seat. The participation of each interest group is necessary. Contributions and originality: support in the management of the Comprehensive Tourism Development Plan, identification of strengths and opportunities in natural, gastronomic and cultural resources, training to improve the quality of services and products in communities with little developed tourism potential such as Oyameles and Mazatepec.

Keywords: development; economy and business; Magic Town; population; quality, tourism.

JEL codes: Z32 Tourism and development 


\section{Introducción}

El objetivo fue conocer los resultados del programa desde la percepción de los grupos de interés: los empresarios, la ciudadanía, los turistas y las autoridades municipales, quienes han contribuido a mejorar las condiciones de empleo, económicas y sociales. La justificación de este estudio ubica al turismo como parte de la actividad económica relevante para mejorar la calidad de vida de la población de las comunidades con potencial turístico, a través de la generación de empleos, la difusión de la cultura, las tradiciones y la biodiversidad que poseen los 132 municipios con el distintivo de Pueblo Mágico Sectur, 2020). En este contexto, según Torruco (2021), el crecimiento del producto interno bruto (PIB) en el rubro turístico a finales del año 2021 será del 7,1 \% en relación con el PIB nacional, con una estimación de 31 millones de visitantes extranjeros, que representará una derrama económica de 18100 millones de dólares, con el $46 \%$ de ocupación hotelera. En el presente trabajo se realiza una aproximación del impacto de los resultados del Programa Pueblos Mágicos, implementado por el Gobierno Federal mexicano, tomando como referencia el municipio de Tlatlauquitepec desde la obtención del distintivo hasta la fecha. En cuanto a la problemática, la riqueza natural, cultural, histórica y gastronómica con la que cuenta México es una oportunidad para la reactivación económica y ha sido identificada por la Secretaría de Turismo (Sectur). A partir de 2001 se crea el Programa de Pueblos Mágicos con la finalidad de promover el desarrollo local de las 132 regiones reconocidas hasta hoy con dicha denominación. Entre ellas se encuentra el Pueblo Mágico de Tlatlauquitepec, que se ubica entre los diez mejores atractivos turísticos del estado de Puebla. Este es un punto de referencia para los turistas nacionales y extranjeros que aprecian la naturaleza, la cultura y la gastronomía. En la organización de este artículo se describen las principales características, como actividades económicas y organización política. Así mismo, se plantean diferentes enfoques sobre el programa, se explica la metodología, el diseño de la muestra, los instrumentos utilizados para el levantamiento de la información de campo y el proceso y análisis de la información. Con base en los principales resultados se concluye que el distintivo es una marca que en los últimos años se ha posicionado en la mente de los turistas y está dirigido a un segmento de la población con preferencias por la naturaleza, las costumbres, las tradiciones y la cultura gastronómica. Lo que se tiene claro es que este programa federal ha destinado recursos financieros y materiales para su operación solo en el primer año, período en el que se busca acondicionar los espacios y con ello alcanzar los objetivos. Para la operación del programa, los responsables son las autoridades municipales en coordinación con un equipo de ciudadanos, Ilamado Comité, según lo establece el manual de operación. Sin embargo, en la práctica no se aplica porque el distintivo se convierte en un documento de adorno y de publicidad para el municipio, aspecto que 
no se conoce y que evidencia la necesidad de que en un futuro próximo se haga investigación aplicada sobre las acciones estratégicas para coordinar los esfuerzos de los diferentes grupos de interés. Los aportes de esta investigación servirán de apoyo a las autoridades municipales en la gestión del Plan de Desarrollo Turístico Integral, así como a las partes interesadas para identificar las fortalezas y oportunidades de los recursos naturales, gastronómicos y culturales.

\section{Antecedentes del Pueblo Mágico}

A principios del siglo XVI, Tlatlauquitepec fue habitado por pequeños grupos olmecas. Posteriormente, se asentaron grupos toltecas y durante la expansión del Imperio azteca llegaron los chichimecas, quienes se trasladaron a las montañas para no ser sometidos al reinado de Tenochtitlán (Sectur, 2014).

Fue sede del señorío prehispánico y controlaba los tributos que entregaban varios pueblos de la región cada 80 días a los mexicas. Además, los franciscanos provenientes del estado de Tlaxcala se encargaron de la evangelización del lugar y se formó el convento de Santa María Tlatlauquitepec. Otros sitios cercanos en 1526 crearon las casas conventuales en Tlatlauquitepec en 1531, Hueyapan en 1535 e Ixtacamaxtitlan en 1548. Tlatlauquitepec fue el punto de partida para llevar el evangelio a todos los pueblos de la sierra y de la costa (Instituto Nacional para el Federalismo y el Desarrollo Municipal [INAFED], 2010).
A través de la historia puede observarse que Tlatlauquitepec ha mantenido una posición política, económica y cultural en el desarrollo de la región nororiental de Puebla, parte de ello evidenciado en la figura 1, lo que dio como resultado que el 28 de noviembre de 2012 fuera declarado Pueblo Mágico. En la figura 2 se muestran las letras monumentales que identifican este destino turístico.

Figura 1. Zócalo de Tlatlauquitepec, Puebla.

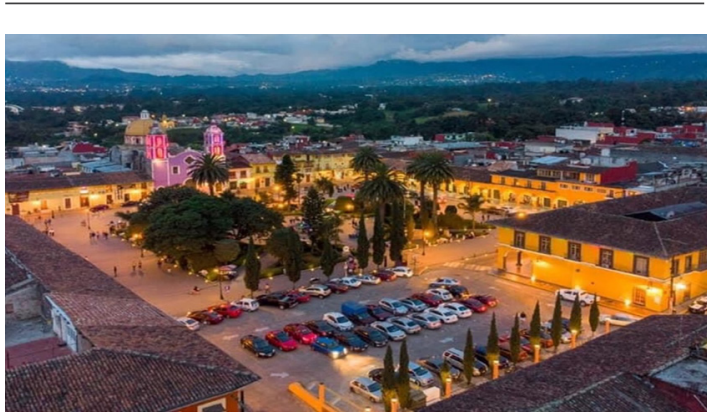

Fuente: fotografía tomada durante el trabajo de campo.

Figura 2. Tlatlauquitepec, Pueblo Mágico.

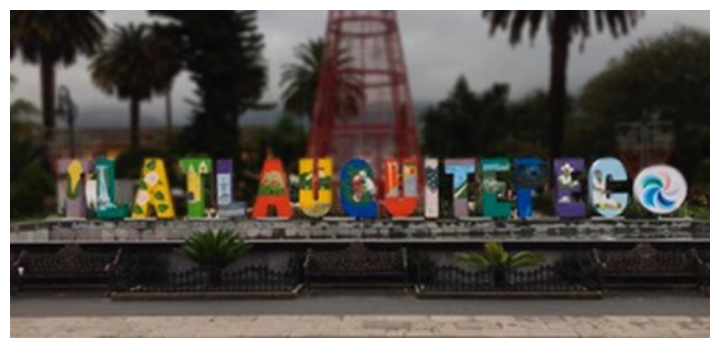

Fuente: fotografía tomada durante el trabajo de campo.

\section{Medio físico}

El municipio de Tlatlauquitepec, Puebla, se encuentra localizado en los paralelos $19^{\circ} 36^{\prime} 24^{\prime \prime}$ y $20^{\circ} 03^{\prime} 18^{\prime \prime}$ de latitud norte y en los meridianos $97^{\circ}$ $14^{\prime} 42^{\prime \prime}$ y $97^{\circ} 28^{\prime} 06^{\prime \prime}$ de longitud occi- 
dental. Cuenta con una altitud mínima de 300 msnm y una máxima de 2900 msnm (Ayuntamiento de Tlatlauquitepec, 2018).

Como se muestra en la figura 3, Tlatlauquitepec está situado a $130 \mathrm{~km}$ de la ciudad de Puebla, en la parte noreste del estado, y colinda con 11 mu- nicipios: al norte, con Cuetzalan del Progreso y Ayotoxco de Guerrero; al este, con Hueyapan, Yaonáhuac, Tételes de Ávila Castillo, Atempan y Chignautla; al sur, con Cuyoaco y al oeste, con Zautla, Zaragoza y Zacapoaxtla.

Ocupa el cuadragésimo lugar de mayor extensión entre los 217 muni-

Figura 3. Colindancias de Tlatlauquitepec.

Prontuario de información geográfica municipal de los Estados Unidos Mexicanos Thatlauguitepec. Puebla

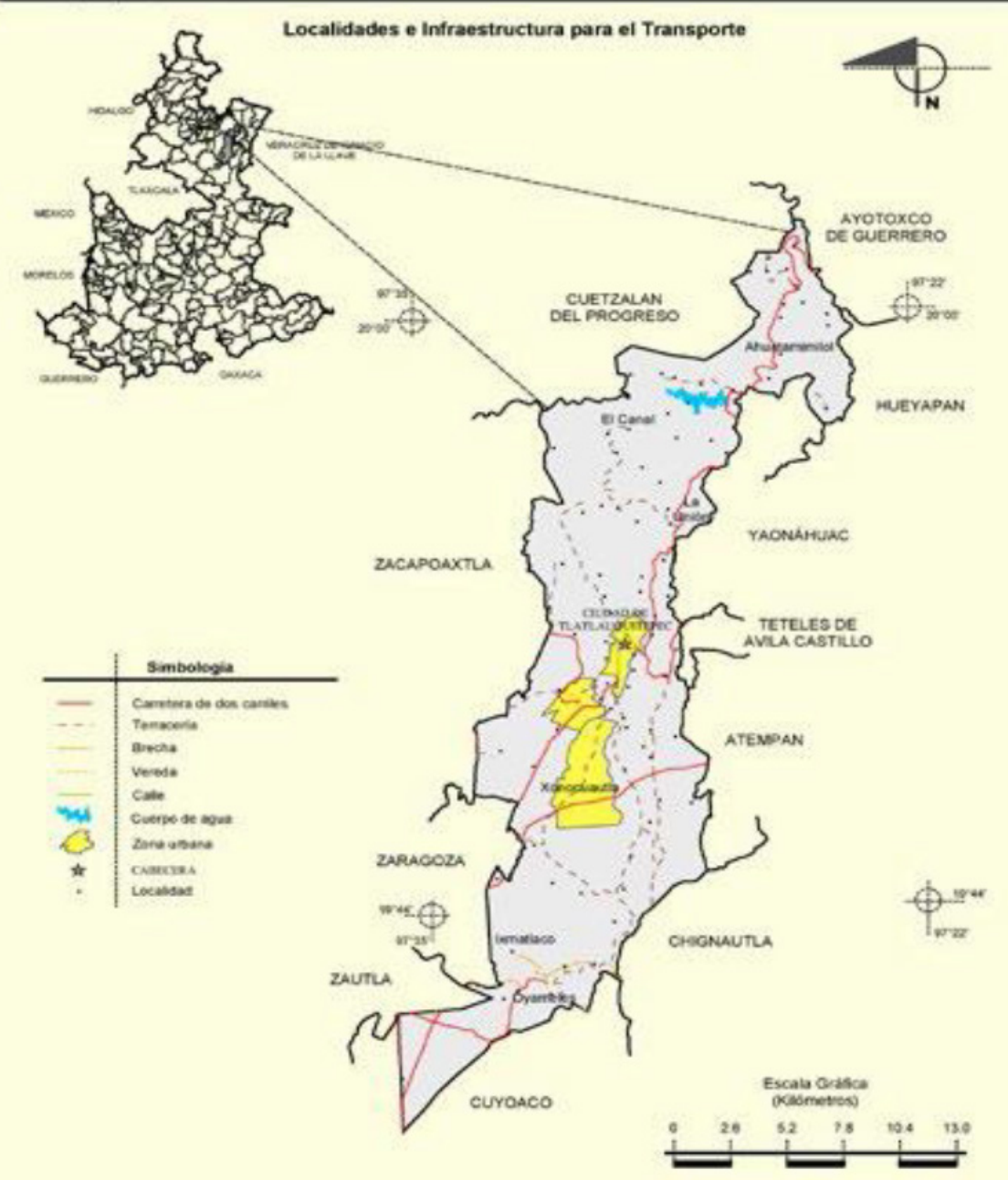

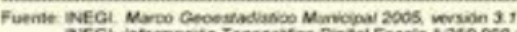

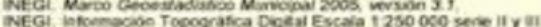

Fuente: tomado del Plan Municipal de Desarrollo, (Ayuntamiento de Tlatlauquitepec, 2018). 
cipios que conforman el estado de Puebla, con 296,15 km², que representan el 0,9\% del territorio estatal.

Los ríos que pasan por Tlatlauquitepec se originan en la zona sur del municipio, hacen su recorrido por la parte norte y se concentran y desembocan en el río Apulco. Su recorrido culmina en la presa hidroeléctrica La Soledad, conocida también como Presa de Mazatepec. Cabe mencionar que el recorrido de los ríos por Tlatlauquitepec se da hasta que se topan con elevaciones entre San Agustín Chagchaltzin y El Progreso, donde sus cauces se desvían hacia el oriente y se concentran fuera del municipio en el río Xucayucan, el cual está conformado por los ríos Atemeza, Xochihuatzaloyan y Atemochicha.

Tlatlauquitepec cuenta con una gran variedad de climas, con la transición de la Sierra Norte y el declive del golfo de México, tales como semifrío subhúmedo con lluvias en verano en las montañas del sureste, templado subhúmedo con lluvias en verano al sur, templado húmedo con Iluvia abundante en verano en la parte central y templado húmedo con Iluvias todo el año en la parte central.

Al norte del municipio se encuentra un bosque abundante mesófilo de montaña conformado por liquidámbar y jaboncillo. Por su parte, en la zona central y sur se cuenta con áreas reducidas de pino, encino y oyamel. Aunque predomina el pino colorado, el roble y el oyamel, también hay pastizales adaptados al bosque.

Entre las especies de fauna se encuentran conejos, ardillas, tuzas, armadi- llos, variedad de reptiles y de aves. Infortunadamente, a consecuencia de la caza y los desmontes, diversas especies se extinguieron, entre ellas el venado temazate y el pumilla o tigrillo. Se conoce como el Jardín de la Sierra Norte, por sus verdes paisajes.

\section{Características de la población}

De acuerdo con el Anuario estadístico de Puebla, la población total de Tlatlauquitepec es de 53.498 habitantes, de los cuales el 46,7\% corresponde a hombres y el 53,3\% a mujeres (INEGI, 2017). Además, cuenta con 91 localidades distribuidas por rango de habitantes, como se indica en la tabla 1.

Tabla 1.Distribución de localidades por rango de habitantes

\begin{tabular}{c|c|c}
$\begin{array}{c}\text { Número de } \\
\text { localidades }\end{array}$ & $\begin{array}{c}\text { Rango de } \\
\text { habitantes } \\
\text { por localidad }\end{array}$ & $\begin{array}{c}\text { Ubicación por- } \\
\text { centual en rela- } \\
\text { ción con el total }\end{array}$ \\
\hline 20 & Menor a 100 & $1,6 \%$ \\
\hline 43 & 101 a 500 & $20,8 \%$ \\
\hline 20 & 501 a 1500 & $28,8 \%$ \\
\hline 8 & 1501 a 10.000 & $48,8 \%$ \\
91 & & $100,0 \%$ \\
\hline
\end{tabular}

Fuente: elaboración propia con datos tomados del Plan Municipal de Desarrollo (Ayunyamiento de Tlatlauquitepec, 2018).

\section{Cultura}

Tlatlauquitepec tiene un museo etnográfico particular con más de 1700 piezas, conformado por un gran patrimonio cultural, de fotografías, madera, herrería y cristalería que, en alguna época, fueron parte de la vida económica, patrimonial y cultural.

En su gastronomía se encuentran tlayoyos rellenos de fríjol, alverjón, papa 
y haba; pollo y carnes ahumadas, chayoteste, café, vinos tradicionales, mole ranchero y dulces típicos, algunos de ellos mostrados en la figura 4.

Figura 4. Gastronomía, dulces típicos y vino de Tlatlauquitepec.
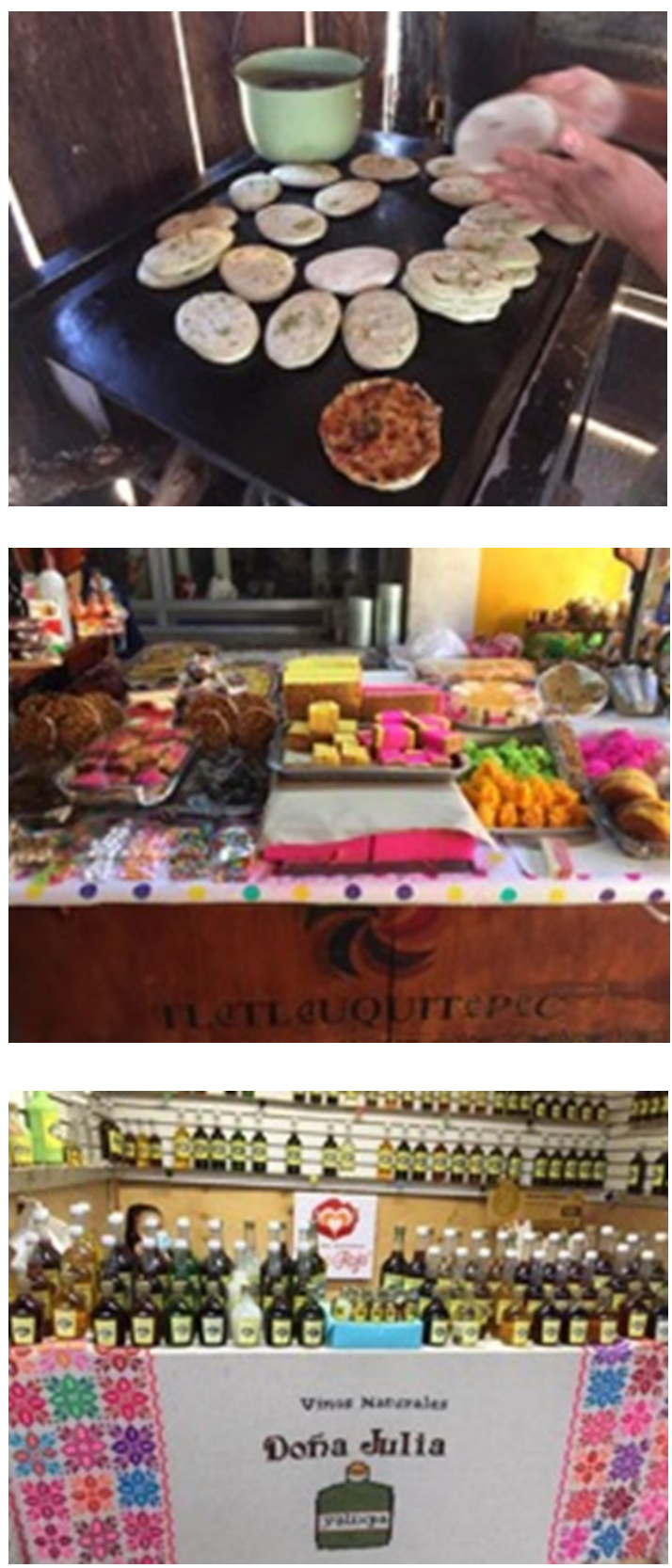

Fuente: fotografía tomada durante el trabajo de campo.
Cada año, en el mes de marzo, se lleva a cabo el Festival del Cerro Rojo. El municipio también ofrece actividades artísticas y culturales, como el encuentro de productores de tunas y danzas autóctonas, además de la veneración de la Virgen de la Asunción, el 15 de agosto, y el concurso de la flor tuberosa.

Entre sus monumentos históricos se encuentran la plaza principal, el exconvento de Santa María de la Asunción, el Santuario del Señor de Huaxtla y, como se muestra en la figura 5, la Iglesia del Sagrado Corazón de Jesús (Sectur, 2019).

Figura 5. Iglesia del Sagrado Corazón de Jesús.

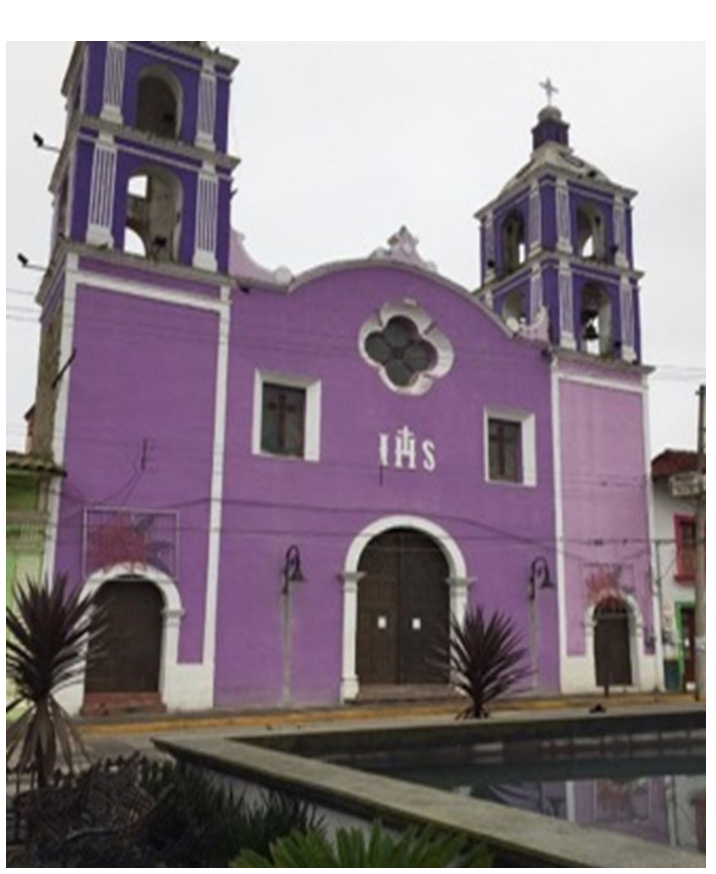

Fuente: fotografía tomada durante el trabajo de campo.

\section{Servicios públicos}

El $97 \%$ de la población cuenta con agua entubada y el $3 \%$ tiene acceso 
por acarreo. El 96,81\% del total de la población dispone de energía eléctrica y el resto no. Con relación a la disposición del drenaje, el 56,01 \% de las viviendas está conectado a la red pública, el 39,77 \% se conecta a una fosa séptica, el 3,34 \% descarga en un río o lago y el 0,87\% dispone de barranca o grieta (Ayuntamiento de Tlatlauquitepec, 2018).

Con respecto a la educación, el $98,6 \%$ de las personas con edad de 15 a 24 años sabe leer y escribir. Sin embargo, en las personas de 25 años y más este porcentaje es del 83,4 \%. Finalmente, en relación con el servicio de salud, el 12,4 \% de la población no tiene acceso. Del total de la población de derechohabientes, el 85,2 \% está afiliado al seguro popular, el 5,7\% al Instituto Mexicano del Seguro Social (IMSS), el $9 \%$ al Instituto de Seguridad y Servicios Sociales para los Trabajadores del Estado (ISSSTE) y el resto a Petróleos Mexicanos, a la Defensa Nacional o Marina y al seguro privado.

\section{Economía}

Este rubro incluye todas las actividades productivas que realizan los habitantes del municipio, donde el 40,9\% pertenece al sector de la población económicamente activa (PEA), conformada por un 29,1\% de mujeres y un 70,9\% de hombres (Ayuntamiento de Tlatlauquitepec, 2018).

Del total de la PEA, el 96,8\% está ocupado. El 38,4 \% de la población ocupada se encuentra en el sector primario, el 16,5\% en el secundario y el 43,2\% en el terciario, con un 11,7\% representado por la industria comercial. El municipio registra 1421 unidades económicas, con tres sucursales de banca múltiple (Ayuntamiento de Tlatlauquitepec, 2018).

Actualmente, cuenta con 16 hoteles y 231 habitaciones, con un promedio de estancia de 1,45 noches. La derrama en 2020 fue de 12.194.294 pesos. Sin embargo, hubo una disminución del 65 \% con relación a los ingresos obtenidos en 2019, que fueron de 34.747.862 pesos, debido al confinamiento por la pandemia del COVID-19 (Gobierno de Puebla, 2020: Sectur Puebla, 2021).

\section{Organización política}

El municipio de Tlatlauquitepec está conformado por un ayuntamiento, que lo integran un presidente municipal, un síndico y ocho regidores, de los cuales seis son de mayoría relativa y dos de representación proporcional. (INAFED, 2010). El Honorable Ayuntamiento tiene las siguientes comisiones: gobernación, justicia y protección civil; desarrollo urbano, ecología y medio ambiente, obras y servicios públicos; industria, comercio, agricultura y ganadería; grupos vulnerables, personas con discapacidad, juventud y equidad de género; salubridad y asistencia pública; turismo; patrimonio y hacienda pública; educación pública, actividades culturales, deportivas y sociales.

El estudio y despacho de la Administración Pública Municipal Centralizada 
está conformada por la Presidencia Municipal, la Secretaría del Ayuntamiento, la Tesorería y la Contraloría municipal (Ayuntamiento de Tlatlauquitepec, 2019). Cuenta con cuatro juntas auxiliares: Mazatepec, Ocotlán de Betancourt, Oyameles de Hidalgo y Xonocuautla, las cuales son administradas por un presidente auxiliar y cuatro integrantes propietarios.

\section{Contexto teórico}

En cuanto al turismo, en diciembre de 2020, México ocupó el tercer lugar en ser visitado y el décimo cuarto en captación de divisas turísticas (Forbes, 2020), lo que significa que el sector turismo forma parte de las principales actividades económicas del país. En este contexto, el Programa Pueblos Mágicos es uno de los mejores posicionados, ya que, desde su creación en el año 2001, a pesar de la alternancia de tres partidos políticos diferentes durante tres sexenios en el Gobierno de México, continúa en operación con gran aceptación y se han adicionado poco a poco diferentes comunidades hasta sumar 132 Pueblos Mágicos a nivel nacional (Entorno Turístico, 2021). El distintivo representa una posibilidad de reactivación económica en la localidad, aprovechando los recursos naturales, históricos y gastronómicos con los que cuenta. Al respecto, con base en los estudios realizados, Acerenza (2013) señala que el desarrollo del turismo a nivel local incentiva el crecimiento económico y fomenta la protección y el cuidado de los recursos naturales y culturales; además, potencializa el turismo regional y nacional con el objetivo de mejorar la calidad de vida de la población.

Un Pueblo Mágico es una localidad que tiene atributos simbólicos, leyendas, historia, hechos trascendentes, cotidianidad; es decir, magia que emana en cada una de sus manifestaciones socioculturales, las cuales representan una oportunidad para aprovechar el turístico (Sectur, 2016).

El Programa Pueblos Mágicos es una política gubernamental creada por la Secretaría de Turismo, que busca la valoración de las riquezas tangibles e intangibles de los pueblos mexicanos y la creación de identidad a través de estos (Pérez, 2018, p. 21). Así mismo, de acuerdo con Rojas (2015), la magia que caracteriza a los Pueblos Mágicos se debe a la cultura indígena y colonial.

El distintivo otorga autenticidad, entendida como el factor esencial que integra los valores únicos, genuinos y auténticos de Tlatlauquitepec, que lo fortalecen a través del patrimonio con el que cuentan sus comunidades para su aprovechamiento turístico sostenible.

Todas sus localidades cuentan con un inventario actualizado de los recursos y atractivos naturales, culturales y gastronómicos, así como con un directorio actualizado de los prestadores de servicios turísticos. Dicha información es importante para integrar el diagnóstico de las condiciones socioeconómicas, culturales y ambientales a nivel municipal y, de este modo, establecer la pauta para el diseño del Programa Municipal Integral de Turismo. 
Con respecto al financiamiento destinado al Programa de Desarrollo Regional Turístico Sustentable y Pueblos Mágicos, para el ejercicio fiscal 2021 fueron autorizados 4715,13 millones de pesos (Gaceta del Senado, 2020). La mayoría de los recursos tiene como destino la construcción o mejora de la infraestructura turística y el mejoramiento de la imagen del centro histórico de los Pueblos Mágicos, con la finalidad de reactivar la economía y desarrollar el potencial turístico (Secretaría de Gobernación, 2019), además de fomentar la promoción y publicidad que otorga el distintivo.

En el año 2020 se otorgaron once nombramientos más al Programa Pueblos Mágicos, por lo que, actualmente, suman 132. Esto significa que tanto el Gobierno federal como estatal deben considerar un incremento en el presupuesto destinado a dicho programa. De acuerdo con Armenta (2016), al incrementar la cantidad de comunidades, se reduce el nivel de presupuesto para la operación del programa en cada pueblo, ya que se demanda una mayor cantidad de recursos para mejorar las condiciones de la infraestructura en los destinos, con foco en las cabeceras municipales.

En los últimos años, el sector turístico ha generado recursos económicos para las comunidades poblanas. Los ingresos que generó en Puebla, durante 2019, alcanzaron los 15.853.000.000 de pesos, con un incremento del 6,7\% con respecto al año anterior. Cabe mencionar que, del total señalado, el $15 \%$ fue generado por los Pueblos Mágicos (Sectur
Puebla, 2021). Con este comportamiento se evidencia que el desarrollo económico en los Pueblos Mágicos de Puebla todavía es desigual, ya que existe una gran concentración de turistas en Puebla capital, San Andrés, San Pedro Cholula y Tehuacán, destinos con mejores condiciones de infraestructura por su ubicación y posicionamiento regional, lo que les permite captar y recibir mayor cantidad de apoyos de parte del Gobierno federal y estatal.

El turismo se ha convertido en la plataforma para el desarrollo del país, debido a que el programa puede reactivar actividades productivas al generar empleo y oportunidades de negocio e impulsar la renovación integral de las comunidades, entre las cuales se ubican las empresas dedicadas al hospedaje y alojamiento, así como los comercios y negocios de alimentos para consolidar al turismo como motor de crecimiento y desarrollo sustentable (Sectur, 2018).

Aunado a ello se debe ofrecer capacitación y profesionalización a los prestadores de servicios turísticos y productores de artesanías, con la finalidad de mejorar constantemente la calidad en el servicio turístico que asegure la satisfacción del cliente y sea la mejor estrategia para promocionar el lugar de destino.

Todas las estrategias antes mencionadas tienen como finalidad cumplir con los objetivos del programa a través de acciones de responsabilidad social que permitan mejorar la calidad de vida. De acuerdo con Linton 
(citado en Ninco \& Duque, 2017, p. 169-170), hay tres enfoques que las empresas deben tener en cuenta para alcanzar objetivos económicos: los negocios pueden encontrar los mejores métodos para hacer dinero, deben identificar la forma de funcionar incorporando la ética en la producción y deben encontrar la mejor manera de que el modelo creado por el programa funcione al generar valor social y desarrollo.

Por su parte, García y Guerrero (2014, p. 78-79) señalan que el éxito o fracaso dependerá de la interacción y los acuerdos entre los distintos actores involucrados localmente y la participación que el Gobierno federal tiene en la promoción y coordinación en materia presupuestal, en colaboración con los gobiernos estatales y municipales. Sin embargo, para la operación del programa y el cumplimiento de los objetivos se requiere una gran cantidad de recursos para acondicionar los lugares turísticos destino y no solo otorgar el distintivo en serie. El Programa Pueblos Mágicos debe tener continuidad entre los diferentes gobiernos sin importar las preferencias políticas.

\section{Materiales y métodos}

Para la realización de este trabajo se utilizó el enfoque de investigación mixto (cuantitativo-cualitativo) descriptivo, el cual trata de explicar el fenómeno de estudio desde las carac- terísticas más importantes (Munch \& Ernesto, 2019) del Pueblo Mágico de Tlatlauquitepec, para lo cual se identificó la población. Posteriormente, se ubicaron cuatro segmentos para abordar el estudio: a) autoridades; b) ciudadanos; c) visitantes y d) empresarios. El objetivo de ello fue integrar una investigación completa para su análisis e interpretación de resultados (Hernández et al., 2014).

Para determinar las muestras, primero se realizó la investigación documental en el Instituto Nacional de Estadística y Geografía (INEGI), el Directorio Estadístico Nacional de Unidades Económicas (DENUE) y Sectur, entre 2019 y 2020. Se calculó el tamaño de cada una de las cuatro muestras con un nivel de confianza del $95 \%$ y error de estimación de $5 \%$ para poblaciones finitas, como se muestra en la tabla 2.

Para levantar la información se diseñaron cuatro cuestionarios que se aplicaron en campo para cada una de las poblaciones en estudio. Esto se complementó con entrevistas a informantes clave de la comunidad y se apoyó con la observación.

Posteriormente, se revisó la información obtenida en campo, se procesó y se analizaron los datos en el programa Statistical Package for the Social Sciences (SPSS), aplicando el método descriptivo correlacional para el análisis de las variables 
Luz del Carmen Marán-Brava; Ricardo Dsorio-Gómez; María de los Ángeles Flores-Aguilar; Héctor De Sampedro-Pablano

Tabla 2.Distribución de la muestra para las cuatro poblaciones

\begin{tabular}{|c|c|c|c|}
\hline Segmento & Características & $\begin{array}{l}\text { Tamaño } \\
\text { muestra }\end{array}$ & $\begin{array}{c}\text { Ítems por } \\
\text { instrumento }\end{array}$ \\
\hline Autoridades & $\begin{array}{l}\text { Se trata de un municipio pequeño y cuenta con una estructura } \\
\text { básica (regidores, síndico y presidente municipal). Se eligieron } \\
\text { autoridades cuyos puestos estuvieran directamente relaciona- } \\
\text { dos con el programa, el turismo y la economía. }\end{array}$ & 6 & 12 \\
\hline Ciudadanos & Habitantes mayores de 18 años, residentes del Pueblo Mágico. & 382 & 21 \\
\hline $\begin{array}{l}\text { Turistas / } \\
\text { visitantes }\end{array}$ & $\begin{array}{l}\text { Turistas locales, nacionales o extranjeros que visitan el Pue- } \\
\text { blo Mágico. Se calculó el promedio anual, posteriormente, se } \\
\text { calculó un promedio mensual y, finalmente, un promedio dia- } \\
\text { rio, para lo cual se estableció la base promedio de } 15 \text { días de } \\
\text { afluencia entre fines de semana y festividades específicas del } \\
\text { Pueblo Mágico. }\end{array}$ & 23 & 16 \\
\hline Empresarios & $\begin{array}{l}\text { Encargados, dueños, gerentes de empresas y negocios rela- } \\
\text { cionados con la actividad turística (hoteles, hostales, moteles, } \\
\text { restaurantes, fondas, tiendas de artesanías, servicios de trans- } \\
\text { porte, comercios). }\end{array}$ & 38 & 34 \\
\hline
\end{tabular}

Fuente: elaboración propia a partir de la información obtenida de INEGI, DENUE y Sectur (2019-2020)..

\section{Resultados}

Para analizar la información obtenida en campo, esta se organizó por grupos de actores: autoridades, pobladores de la comunidad, visitantes y empresarios. En una segunda etapa se integró en un grupo para el análisis, lo que dio como resultado una investigación completa.

\section{Autoridades}

Como se muestra en la figura 6, las autoridades del municipio coinciden en que las actividades relacionadas con el comercio al por mayor y al menudeo son una fuente de ingresos monetarios importante para la población, con un $50 \%$. Esto se complementa con las actividades relacionadas con la agricultura, ganadería, forestación y pesca, con el 33 \% y después se ubica el turismo, con el $17 \%$. Al analizar la variable de empleo, se observa que los servicios producidos por el comercio son los que generan mayor cantidad, seguido por las actividades del sector primario y, por último, se ubican las actividades generadas por el turismo. Cabe señalar que la mayor generación de empleos en la cabecera municipal se da por temporadas, cuando hay afluencia de turistas.

Desde el punto de vista de las autoridades, el 50 \% coincide en afirmar que desde la denominación como Pueblo Mágico, en 2012, se han desarrollado otras actividades económicas asociadas al turismo, como nuevas rutas en el transporte por la apertura de atractivos naturales, creación de nuevos comercios en un $30 \%$, construcción de nueve hoteles más en la cabecera municipal, dos en la junta auxiliar de Mazatepec y uno en Ocotlán, además de visitas guiadas para realizar activi- 
dades de senderismo, a campo traviesa, visita a las luciérnagas, eventos gastronómicos en la cabecera municipal y en las juntas auxiliares, entre ellos la Feria del Itacate en la comunidad de Mazatepec.

Figura 6. Actividad económica que genera más ingresos monetarios a la comunidad.

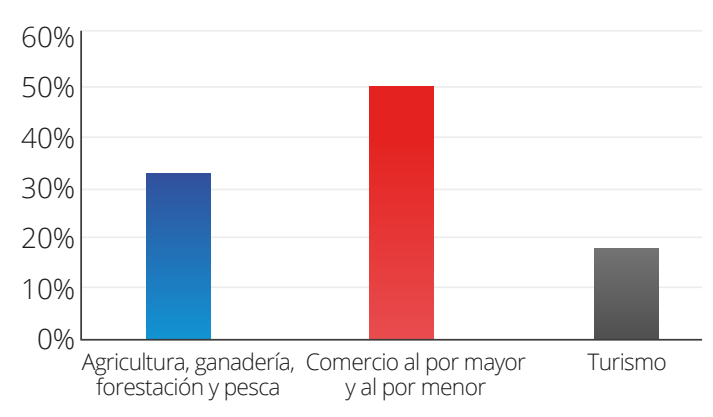

Fuente: elaboración propia con datos de campo, 2020.

Sin embargo, según las autoridades, la apertura del turismo en el municipio y en las juntas auxiliares ha provocado diversos cambios paulatinos en el comportamiento de la población joven respecto a su cultura, costumbres y tradiciones en un $50 \%$; por el contrario, en cuanto a la forma de organización de la comunidad e integración de la economía doméstica familiar, no se identifican cambios en el 50 \%.

Con respecto al tema del mejoramiento de la infraestructura y los servicios de la comunidad, el $67 \%$ dice que se realizaron mejoras parciales en la pavimentación de las calles, mantenimiento de los caminos rurales y carreteras que comunican con las comunidades que tienen algún atractivo turístico, así como espacios públicos, plazas y centros ceremoniales, como el Cerro Cabezón o Cerro Rojo, entre otros.
También se preguntó a las autoridades acerca del apoyo y la inversión pública en el sector turismo. El 67 \% dijo que la inversión es casi nula. En un principio, cuando otorgaron el distintivo, fue menor del $20 \%$ del total del presupuesto asignado al municipio y, posteriormente, el Gobierno solo apoyó con la publicidad, anunciando la marca de Pueblo Mágico. Actualmente, este programa no cuenta con una partida específica para operar, por lo que usa el gasto corriente del municipio. Cabe señalar que con la pandemia el presupuesto asignado al municipio se redujo y a ello se le suma la casi nula afluencia del turismo en las comunidades de Tlatlauquitepec.

Las autoridades reconocen que contar con el distintivo de Pueblo Mágico le ha dado publicidad a Tlatlauquitepec, pero no perciben un beneficio directo para lograr apoyos, adquirir recursos financieros para proyectos turísticos nuevos o mantener los que ya se tienen. Tampoco hay trato especial para obtener apoyos financieros o materiales de programas federales o estatales que fomenten las actividades turísticas de forma sustentable y con ello la reactivación económica local de las comunidades.

\section{Ciudadanos}

Los pobladores de la comunidad dicen que la principal actividad económica es la agricultura y la ganadería, con un 51 \% (figura 7), pues en ella basan su alimentación y sobrevivencia; le sigue el comercio y la prestación de servicios, con el $39 \%$ y el turismo, con el $5 \%$. Cabe señalar que, en gran 
parte, los servicios operan por la actividad turística; sin embargo, las personas no lo consideran como parte importante de su economía, lo que se observa es que la población está en formación para valorar el turismo.

El $75 \%$ de la población reconoce que a partir de 2012, con la denominación de Pueblo Mágico, la afluencia del turismo se incrementó (figura 8), por lo que fue necesario crear espacios para hospedaje. Así mismo, se diversificó el comercio y se incrementó el número de comerciantes locales y de comunidades aledañas a la cabecera municipal. Los pobladores señalan que los períodos con mayor concurrencia de turistas se presentan en los meses de marzo, agosto, septiembre, noviembre y diciembre, para visitar sus atractivos naturales, la Iglesia del Sagrado Corazón de Jesús, el Santuario del Señor de Huaxtla, la presa de La Soledad, la cascada de Puxtla, la cascada de Tenazate, los manantiales de Xiliaco y el cultivo de orquídeas.
Esto obedece a las festividades, eventos culturales, religiosos y naturales, como el Festival del Cerro Rojo y el encuentro de productores de tunas. En su visita, los turistas veneran a la Virgen de la Asunción, realizan pastorelas, danzas, cabalgatas, avistamiento de luciérnagas, asisten a las fiestas del Día de Muertos, a la carrera de relevos de 80 kilómetros, a las callejoneadas y leyendas, al festival en honor a la flor tuberosa y las orquídeas, además de conocer paisajes con su riqueza de flora y fauna.

Respecto al interés que muestra la población para contratarse en actividades económicas, el 52 \% de los entrevistados manifestaron su interés por trabajar en el comercio y prestar sus servicios, mientras que el $41 \%$ manifiesta gran interés por seguir trabajando en actividades de la agricultura y la crianza de la ganadería. Le siguen las actividades de la industria y, por último, la pesca, con menor preponderancia.

Figura 7. Actividad principal de la comunidad.

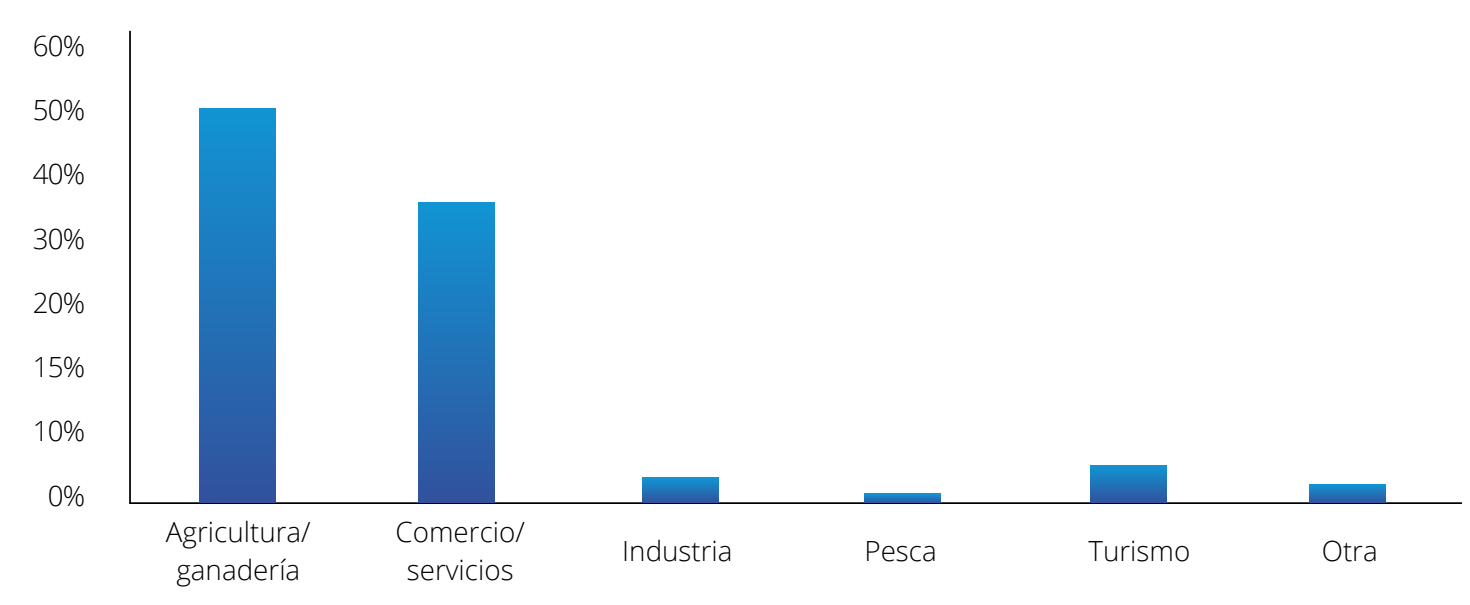

Fuente: elaboración propia con datos de campo, 2020. 
Figura 8. Comportamiento del turismo con la denominación de Pueblo Mágico.

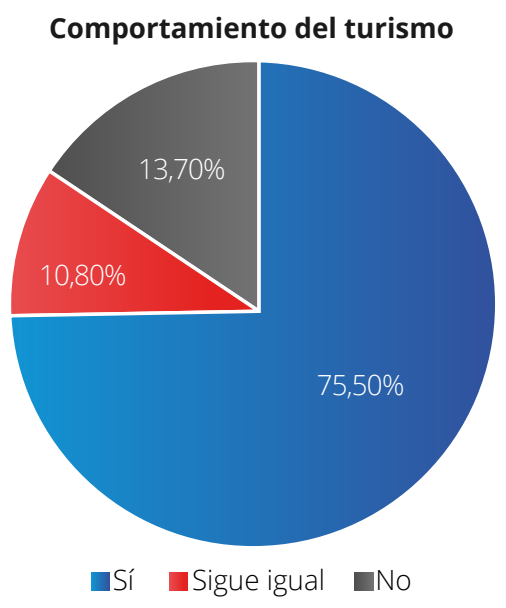

Fuente: elaboración propia con datos de campo, 2020.

Cabe mencionar que la población reconoce parcialmente que la actividad turística es fuente directa generadora de empleo y de ingresos, sobre todo por temporadas. Sin embargo, logra distinguir que existe la apertura de nuevos comercios y que las ganancias se están concentrando en los empresarios y comerciantes, los cuales han incrementado los precios, tanto para los turistas como para los consumidores locales, quienes se ven afectados en la disminución de la capacidad de compra, ya que el dinero les alcanza menos. Además, la afluencia de turistas, de alguna manera, está ocasionando algunos problemas, como la generación de basura, contaminación y deterioro de los recursos naturales. A pesar de que los habitantes valoran grandemente su cultura, tradiciones y recursos naturales, es necesario concientizar a la población y a los visitantes para conservar los ecosistemas y la biodiversidad.

\section{Empresarios}

Desde la opinión de los empresarios y comerciantes, las actividades que generan mayor riqueza son el turismo, los servicios, los restaurantes, bares, cafeterías y el transporte, con el $55 \%$, seguido por el comercio, con el 36 \% y la agricultura, ganadería y forestación, con el $9 \%$, a partir del distintivo de Pueblo Mágico, como se muestra en la figura 9.

Lo anterior significa que el nombramiento de Pueblo Mágico generó un cambio positivo en la economía de la comunidad, beneficiando con mayor impacto a los empresarios y comerciantes.

Figura 9. Actividades económicas que generan mayor riqueza.

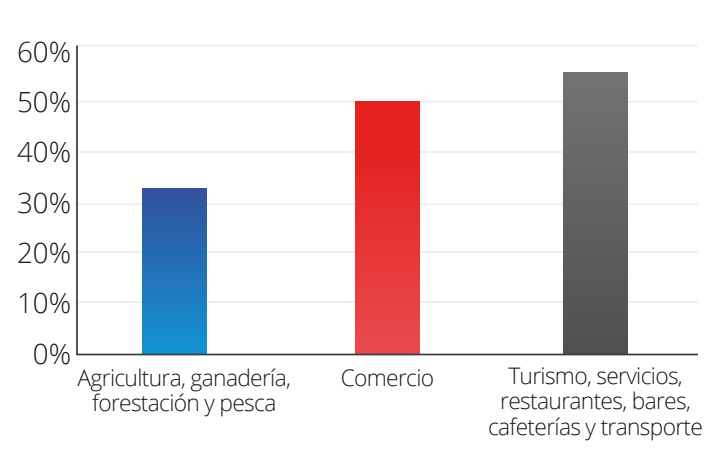

Fuente: elaboración propia con datos de campo, 2020.

\section{Visitantes}

En cuanto a la impresión que tienen los visitantes sobre la calidad y calidez de los servicios que ofrecen en el Pueblo Mágico, el 85 \% las considera buenas y excelentes; el $10 \%$, regulares y el resto, malas (figura 10). Esto indica que, a 8 años de haber recibido el nombramiento, la comunidad poco 
a poco ha mejorado la atención y los servicios que brinda a los turistas. Ello se corrobora con la recomendación que los visitantes hacen, ya que el $95 \%$ de los entrevistados recomiendan visitar el Pueblo Mágico de Tlatlauquitepec.

\section{Discusión}

Con base en la revisión de los resultados, se puede decir que el Programa Pueblos Mágicos, implementado por el Gobierno Federal mexicano, ha fomentado el turismo en 132 pueblos distribuidos a lo largo y ancho de México (Sectur, 2020), se han instalado nuevas empresas y, con ellas, empleos temporales, los cuales no garantizan la estabilidad laboral, puesto que los trabajadores carecen de prestaciones económicas y seguridad social. La ausencia de capacitación formal influye en la calidad de los servicios que se ofrecen.

Al revisar la operación del programa, se identifica que no existe una apropiación de este por parte de la comunidad, ya que se integra el expediente para cumplir con los requisitos que establece la convocatoria y con ello obtener el distintivo. Sin embargo, no se observa un Plan de Desarrollo Turístico Municipal en ejercicio, aunque aparece como requisito normativo. Hay una total ausencia del funcionamiento del comité o grupo de trabajo del Pueblo Mágico, solo se observan los esfuerzos realizados por la dirección de turismo, por parte del Ayuntamiento con los mínimos recursos disponibles por el recorte presupuestal debido a la pandemia del COVID-19. Por su parte, el Gobierno federal y estatal no otorga presupuesto extraordinario para la operación del programa (Armenta, 2016) y tampoco hace seguimiento. Los encargados de la operación señalan que desde la asignación del distintivo no se ha realizado una evaluación para refrendarlo, lo que demuestra un total desinterés por parte de las instituciones federales para asegurar

Figura 10. Calificación de los servicios que ofrece el Pueblo Mágico.

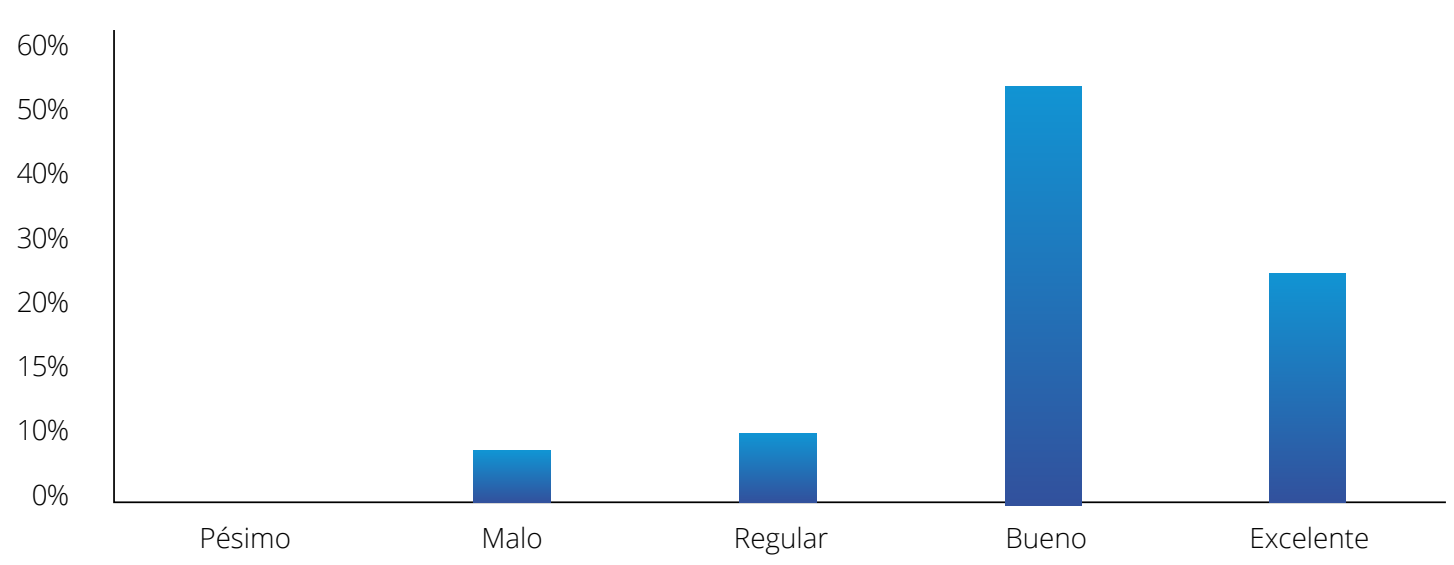

Fuente: elaboración propia con datos de campo, 2020. 
el cumplimiento de los requisitos de la operación del programa.

Se han creado y fortalecido diferentes actividades y productos turísticos, como excursiones, paseos, visitas guiadas y paquetes, con la participación de algunas operadoras turísticas. La dirección de turismo cuenta con un registro de la afluencia de visitantes. En su mayoría se trata de turistas de fin de semana y procedentes de ciudades cercanas como Puebla, Ciudad de México, Veracruz, Querétaro y Oaxaca. Cabe destacar que el turismo se presenta por temporadas, con mayor presencia en los meses de marzo, abril, julio, agosto, noviembre y diciembre.

Dicho programa gubernamental ha contribuido a revalorar e identificar las poblaciones con potencial turístico sin desarrollar, pero que poseen características que han conservado a través del tiempo, entre ellas tradiciones, arquitectura e historia, artesanías, gastronomía, recursos naturales y cultura, las cuales le dan identidad a la comunidad (Pérez, 2018; Rojas, 2015). Tal es el caso de Tlatlauquitepec, que para obtener el distintivo se sometió a una evaluación de autenticidad, lo que le permitió alcanzar el distintivo de Pueblo Mágico en 2012.

La finalidad de esta política gubernamental es reactivar la economía local de los municipios seleccionados a través de una marca registrada a favor de la Secretaría de Turismo, con el propósito de beneficiar a todas las comunidades que se encuentran en la periferia de la cabecera munici- pal. Sin embargo, los resultados de otros estudios han encontrado que la marca por sí misma no garantiza la reactivación de la economía y del turismo, existen otros factores como la infraestructura, las instalaciones, las vías de acceso y la comunicación (Landeros et al., 2015), así como aspectos internos en la forma de organización de la comunidad, la hospitalidad hacia el turista, entre otros, que inciden en el cumplimiento de las metas del programa.

El impacto y los beneficios directos del programa se ubican en los negocios y comerciantes. Por su parte, la ciudadanía no alcanza a percibir los beneficios económicos que el programa pudiera otorgar para mejorar la calidad de vida de las personas y de la comunidad.

Así mismo, no se observa un proyecto turístico a mediano plazo que permita desarrollar el turismo en un esquema de equilibrio entre el manejo de los recursos naturales, el patrimonio cultural, la gastronomía y las tradiciones como un área de oportunidad, para lo cual, según Guerrero y López (2015), se debe hacer un estudio de competitividad mediante un análisis FODA, con la finalidad de hacer propuestas que lleven a la mejora a través de la coordinación entre las autoridades, la población, los empresarios y los visitantes, para así lograr los objetivos que persigue el programa.

\section{Conclusiones}

Con el distintivo de Pueblo Mágico, Tlatlauquitepec ha mejorado paula- 
tinamente la generación de empleos permanentes y temporales, así como la infraestructura, las vías de comunicación y algunos servicios públicos de la cabecera municipal.

Los empresarios relativamente más favorecidos son los que ofertan el servicio de hospedaje y alimentos, así como los comerciantes de productos tradicionales y artesanías de la región. Por el contrario, el poder adquisitivo de la economía familiar se ha visto afectado, debido al incremento en el precio de los bienes y servicios locales.

Es necesaria la participación de todos los actores: autoridades, empresarios, comerciantes, población y turistas para desarrollar comunidades con potencial turístico bajo un enfoque de sustentabilidad.

De manera general, todos los actores estudiados coincidieron en que el turismo ha beneficiado a las comunidades de Tlatlauquitepec. La investigación de campo permitió visualizar diferentes rutas y negocios susceptibles de emprendimiento.

Las autoridades municipales deben poner mayor énfasis y atención en todas las comunidades para fomentar su participación con los productos y servicios que tienen mayor ventaja competitiva y, así, fortalecer la cadena de valor generada por el distintivo o marca, para favorecer los ingresos y mejorar la calidad de vida de todas las comunidades del Pueblo Mágico de Tlatlauquitepec.

En general, el Programa Pueblos Mágicos se ha mantenido constan- te desde su creación el 5 de octubre de 2001 hasta la fecha, a pesar de la alternancia de los gobiernos locales, estatales y federales, por la importancia que representa la actividad turística para el desarrollo local.

\section{Propuestas o contribuciones}

El Programa Pueblos Mágicos es una marca. Por ello, se debe desarrollar y dar seguimiento de forma permanente, evaluando los aspectos y la calidad de los servicios y productos generados en la cadena de valor.

Es necesario que dentro del presupuesto público federal se destine una partida especial para el fortalecimiento de la infraestructura y la imagen de la comunidad, con un esquema de responsabilidad social y sustentabilidad para la conservación de la biodiversidad.

Se deben generar programas de concientización entre la población para fomentar la cultura de atención al turismo, el rescate de la identidad, las costumbres y tradiciones. Así mismo, es necesario aprovechar los talentos y el emprendimiento de los jóvenes, promover el cuidado del medio ambiente y disminuir el consumo masivo de productos contaminantes.

Las instituciones de educación superior de la región deben vincularse con las comunidades y ofrecer capacitación en temas relacionados con el turismo, bajo un esquema de sustentabilidad, es decir, que la actividad turística no sea equivalente de contaminación e invasora de la biodiversidad y que la calidad de los productos 
y servicios que se ofrecen a los visitantes sea alta. Ello permitirá a la población desarrollar nuevos productos y mejorar la calidad de los ya existentes para generar valor agregado que le permita posicionarse a nivel local, regional e internacional.

Fomentar y valorar las actividades tradicionales, como mejores prácticas en el manejo de la agricultura, la ganadería y la pesca, las cuales forman parte de la sustentabilidad y subsistencia de las comunidades y familias ante una situación de pandemia como la que se vive en 2021.

Realizar rutas turísticas temáticas, por ejemplo, del café o de la carne ahumada, formar guías especializados, reactivar los medios de comunicación, como es el tren, poner la señalética para orientar al turista y generar confianza en el visitante con una corresponsabilidad de la comunidad.

Cabe señalar que este trabajo se ha utilizado como referente para desarrollar nuevos proyectos de reactivación económica y turística, como el caso del Proyecto Senderos de Matlalcueyetl, tradición, cultura y naturaleza en San Miguel Canoa Puebla.

\section{Información complementaria}

Agradecemos a la licenciada Fátima Vivas Hernández y al arquitecto Paul de Jesús Soto Martínez por su valiosa colaboración en la aplicación de encuestas y entrevistas con informantes clave. Así mismo, el apoyo de las autoridades del Ayuntamiento de Tlatlauquitepec, en especial a la directora de turismo, licenciada Cristina Romero Villegas, y al cronista de la comunidad, el maestro Ernesto Arrieta Guzmán, que mostraron gran compromiso al proporcionar y facilitar la información. También hacemos un reconocimiento al Sr. Leonel y su familia, a la Organización de Lancheros de la presa La Soledad por el apoyo incondicional y a todas aquellas personas anónimas de las comunidades que ofrecieron su tiempo y conocimiento.

Del mismo modo, reconocemos el apoyo de los alumnos de Servicio Social de la división Tecnologías de la Información de la Universidad Tecnológica de Puebla y a las maestras Verónica Lizardi y Norma Roldan para la captura y procesamiento de la información de las encuestas. A todos ellos, gracias por sus esfuerzos para que este trabajo de investigación pudiera ser realidad.

\section{Referencias}

Acerenza, M. (2013). Desarrollo sostenible

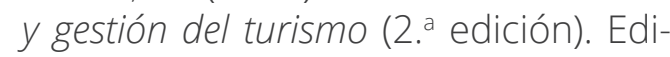
torial Trillas.

Armenta, G. (2016, julio 06). Pueblos Mágicos pierden presupuesto y credibilidad. El financiero. https://bit.ly/3IfAIZe

Ayuntamiento de Tlatlauquitepec. (2018). Plan Municipal de desarrollo Tlatlauquitepec 2018-2021. https://bit.ly/32UwCWa

Ayuntamiento de Tlatlauquitepec. (2019) Manual de organización presidencia municipal. https://bit.ly/3DjYdw

Entorno turístico. Hablemos de México. (2021). Año en que fueron nombrados los 132 pueblos mágicos de México. https://bit.ly/3poikuF 
Forbes. (2020, diciembre 22). México asciende al tercer lugar en recepción de turistas. Forbes México. https://bit.ly/2ZNmiho

Gaceta del Senado. (2020, 18 de noviembre). Proposición con punto de acuerdo por el que el Senado de la República exhorta respetuosamente a la $H$. Cámara de diputados, para que en el análisis, discusión y aprobación del presupuesto de egresos de la federación para el ejercicio fiscal 2021, se asignen y etiqueten recursos al Programa de Desarrollo Regional Turístico Sustentable y Pueblos Mágicos, con el propósito de reactivar la economía de esas localidades. https:// bit.ly/3lqPjag

García-Vega, D. \& Guerrero-García-Rojas; H. (2014). El programa «Pueblos Mágicos»: análisis de los resultados de una consulta local ciudadana. El caso de Cuitzeo, Michoacán, México. Economía y Sociedad, XVIII(31),71-94.

Guerrero, O. \& López, H. (2015) Cadereyta de Montes, Querétaro. Actores del semidesierto. En Pueblos Mágicos: una visión interdisciplinaria. Volumen I. Disponible en https://bit.ly/2ZOq3TT

Hernández-Sampieri, R., Fernández-Collado, C. \& Baptista-Lucio, P. (2014). Metodología de la investigación (6. ta edi- $^{\text {ta }}$ ción). Mc Graw Hill. https://bit.ly/3x$\mathrm{PHOS} 7$

Instituto Nacional de Estadística y Geografía (INEGI). (2017). Anuario estadístico del estado de Puebla 2017. Gobierno del Estado de Puebla. https://bit. ly/3EiR3tr

Instituto Nacional para el Federalismo y el Desarrollo Municipal [INAFED] (2010). Enciclopedia de los municipios y delegaciones de México. https://bit. ly/3sbWF5N
Landeros-Villalva, H., Gómora-Serrano, M. \& Castañeda-Martínez, T. (2015). Enfoques de análisis en Pueblos Mágicos: ¿eficacia o eficiencia? En: Pasado, presente y futuro de las regiones en México y su estudio. Asociación Mexicana de Ciencias para el Desarrollo Regional A.C., México

Münch-Galindo, L. \& Ernesto, A. (2019). Métodos y técnicas de investigación (5. ta edición). Trillas.

Ninco-Hernández, F. A. \& Duque-Orozco, Y. V. (2017). Enfoques de la responsabilidad social empresarial innovadora: alternativas para las organizaciones de hoy. Revista GEON (Gestión, Organizaciones Y Negocios), 4(1), 162-175. https://doi.org/10.22579/23463910.52

Pérez-Towns, A. (2018). La percepción social del programa de Pueblos Mágicos: el caso de Tlatlauquitepec, Puebla [tesis de pregrado, Universidad de las Américas Puebla]. https://bit.ly/3ppzORO

Rojas, C. (2015, octubre 10). Turismo comenzó Programa de Pueblos Mágicos en 2001. Milenio. https://bit.ly/3oiW6oi

Secretaría de Gobernación. (2019). Plan Nacional de Desarrollo 2019-2024. Gobierno de México. https://bit.ly/3mcxE6L

Secretaría de Turismo [Sectur]. (2014) Pueblos Mágicos: Tlatlahuquitepec, Puebla. https://bit.ly/3G3Xms8

Secretaría de Turismo [Sectur]. (2016). Programa de Pueblos Mágicos. Para localidades con atributos simbólicos, historias y leyendas, llenos de hechos trascendentes, cotidianidad y un encanto peculiar. iDescúbrelos! https://bit. ly/31nb1os

Secretaría de Turismo [Sectur]. (2018) Memoria documental Pueblos Mágicos 
(incorporación y permanencia. https:// bit.ly/3DjJjq2

Secretaría de Turismo [Sectur]. (2018) Nuestro Turismo, el gran motor de la economía nacional. https://bit.ly/3lpS9wj

Secrtaria de Turismo [Sectur]. (2019). Tlatlauquitepec, Puebla. Gobierno de México. https://bit.ly/3F8GhG]
Secretaría de Turismo [Sectur]. (2020). Programa de Pueblos Mágicos. Gobierno de México. https://bit.ly/3/1baiD

Secretaría de Turismo [Sectur]. (2021). Estrategia estatal de pueblos mágicos. Gobierno de Puebla. https://bit.ly/3ohQ8Ej

Torruco, M. (2021). México recuperará el PIB turístico prepandemia en 2022: Sectur. Forbes. https://bit.ly/32azzl8 